\title{
NUEVOS SISTEMAS PARA LA EVALUACIÓN CONTÍNUA DEL RENDIMIENTO DEL ALUMNADO. ESTUDIO DE CASO DEL COACHING EN EL GRADO DE INGENIERÍA FORESTAL
}

\author{
Miguel González-Loureiro1: Universidad de Vigo. España \\ mloureiro@uvigo.es
}

\section{RESUMEN}

En este artículo se describe el uso del Coaching para evaluar el rendimiento de los alumnos de forma continua en el área de conocimiento de "organización de empresas" dentro del marco de una titulación de ingeniería como es el Grado en Ingeniería Forestal. En los últimos años el Coaching se está convirtiendo en una herramienta muy útil en el desarrollo profesional y personal. Por eso se incorporó esta nueva herramienta con impactos positivos tanto en la evaluación continua del rendimiento de alumnos como en la transferencia de competencias transversales en estudios universitarios de ingeniería. Así, en el nuevo marco del Espacio Europeo de Educación Superior, el profesor pasa a ser el coach, el guía en el aprendizaje de competencias dejando de lado el rol del docente como "persona que simplemente enseña". Dichas competencias serán aplicadas por el estudiante una vez finalice su etapa en la universidad. Adicionalmente el Coaching como metodología y estrategia docente es una de las más adecuadas para facilitar la evaluación continua del alumno. Así, en el desarrollo profesional y de madurez del alumno se enfatiza el "aprender-haciendo" (learning-by-doing), con la ayuda del coach. Es muy importante en este caso el refuerzo de la función de retroalimentación.

PALABRAS CLAVE: Coaching - Evaluación continua - Grado de Ingeniería Forestal - Organización de empresas - Competencias Transversales 


\title{
NEW SYSTEMS FOR AN ONGOING ASSESSMENT OF THE STUDENTS' PERFORMANCE. CASE STUDY OF COACHING IN THE FOREST ENGINEERING DEGREE
}

\begin{abstract}
This paper describes the use of "Coaching" as a tool to an ongoing assessment of the students' performance, in the knowledge arena of "enterprise organisation", within the framework of the Forestry Engineering Degree. In recent years, coaching is becoming a useful tool in the professional and personal development. Therefore, this new tool was incorporated with positive impacts on both the ongoing assessment of students' performance as well as on the transfer of generic skills, so relevant in university degrees of engineering. Thus, in the new framework of the European Higher Education Area, the professor becomes a coach, a guide in the competences learning process, leaving aside the role of "person who simply teaches". Such competences will be applied by students once they finished their university stage. Furthermore, coaching as methodology and teaching strategy is one of the most suitable to help in the ongoing assessment of the student. Thus, it is emphasised the learning-by-doing in the professional and maturity development of the student, counting on the help of the coach. In this case, strengthening of the role of feedback acquires a high relevance.
\end{abstract}

KEY WORDS: Coaching - Ongoing Assessment of Performance - Forestry Engineering Degree - Enterprise Organisation - Transversal Competences

\section{INTRODUCCIÓN}

\subsection{Organización de empresas. Una asignatura dentro del marco de las ingenierías}

En general, por la carga lectiva de créditos, la docencia impartida por el área de conocimiento de economía de la empresa suele ser minoritaria en las titulaciones de ciencias e ingenierías. Evidentemente, se exceptúa el caso de las ingenierías con la especialidad de organización de empresas.

En el proceso de desarrollo de las guías docentes de una titulación y de cada materia, son fundamentales los métodos de evaluación del rendimiento del alumno. Es importante contemplar cómo las estrategias y metodologías docentes ayudan en la transferencia de las denominadas competencias transversales. Y quizás es aquí, en 
Es un hecho que un porcentaje importante de los alumnos formados en las titulaciones de ingeniería acabará trabajando en el ámbito empresarial ocupando un puesto directivo. De ahí la importancia de entender la perspectiva empresa, sus dinámicas, su cultura, su forma de funcionar, su forma de trabajar. Por lo tanto, son una buena parte de las competencias relacionadas con el saber-estar y el saber-hacer.

En este marco, se presenta el Coaching como una herramienta clave para la evaluación continua y la transferencia de competencias transversales. En este artículo se muestra el caso concreto de su aplicación en la antigua titulación de Ingeniería Técnica Forestal y su posterior adaptación al Grado de Ingeniería Forestal, como innovación docente. En este caso, el Espacio Europeo de Educación Superior (EEES de aquí en adelante) ha facilitado aún más su desarrollo.

Así se espera que el marco del EEES pueda contribuir especialmente a su implantación generalizada en titulaciones en las que el área de conocimiento de economía de la empresa no tiene una presencia relevante, pero sí facilita enormemente el desarrollo de competencias transversales desde una perspectiva más holística.

En los siguientes epígrafes se fundamenta esta herramienta de evaluación del rendimiento del alumno como una innovación docente. Se presenta su aplicación en las titulaciones del plan antiguo de la Ingeniería Técnica Forestal y su adaptación (siendo éste el primer año en que se implanta) al primer curso del nuevo Grado de Ingeniería Forestal.

\section{METODOLOGÍA}

La base metodológica se basa en el análisis de las anteriores escuelas de pensamiento que han tratado el tema que nos ocupa. Se trata de analizar los principales medios existentes hoy y aplicarles un método analítico textual que permita producir inferencias fenomenológicas o estructuras de pensamiento novedoso basado en este tipo de reflexiones que crean un puente entre las ideas anteriores (y sus realidades descritas) y las más modernas

\section{ANÁLISIS Y DISCUSIÓN}

\section{1 Cómo evaluar el rendimiento del alumno en función de la metodología docente empleada}

El nuevo contexto del EEES hace que el concepto enseñanza sufra una profunda 
En este nuevo modelo, los diferentes tipos de competencias van a jugar un papel muy importante. Los alumnos deben adquirir unos hábitos, valores y actitudes, a través de las competencias de ser/estar, como son:

a) Desarrollar actitudes y valores para la participación, así como el trabajo en equipo y colaborativo.

b) Desarrollar actitudes y valores de auto-responsabilidad y autoorganización de su trabajo.

c) Desarrollar capacidades de interrelaciones personales.

d) Desarrollar habilidades de motivación de otras personas.

Y deben desarrollar habilidades y destrezas, a través de las competencias de hacer; tales como:

a) Saber utilizar las técnicas de planificación estratégica: análisis, diagnóstico, diseño de estrategias y retroalimentación.

b) Aprender a analizar y diagnosticar los aspectos internos de la empresa.

c) Aprender a analizar y diagnosticar el entorno de la empresa, para una gestión proactiva.

d) Conocer las bases del diseño de posibles estrategias.

e) Comprender la realidad empresarial del entorno en el que trabajarán

y particularmente de la cadena empresarial de la madera.

f) Saber localizar y utilizar las fuentes de información empresariales.

Para que el profesor pueda ejercer de Coach, es decir, de guía del alumno en su aprendizaje, necesita las herramientas necesarias para evaluar el rendimiento del alumno, centrándose en dos aspectos: la tarea (el proceso) y el resultado final. Así, en el desarrollo profesional y de madurez del alumno, es importante el "aprenderhaciendo" (learning-by-doing). Incluso que el alumno pueda experimentar por sí mismo, escogiendo una de las alternativas que se presenten en cada caso y, con la ayuda del Coach, analizar los errores desde un enfoque didáctico. Por lo tanto, es muy importante el refuerzo de la función de retroalimentación.

No hay un único método docente, ya que según Brown y Atkins (1988) los diferentes métodos de enseñanza pueden ser situados en un continuo. 


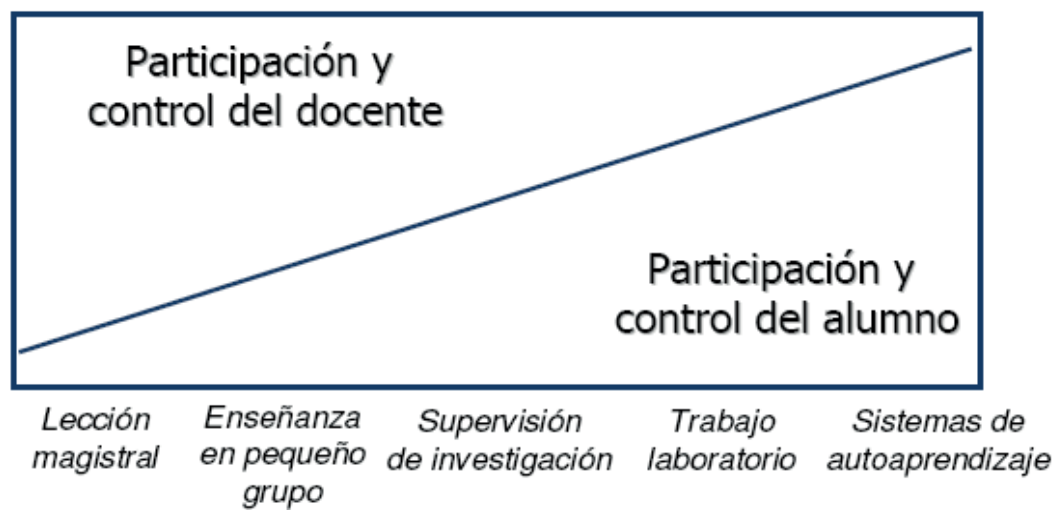

Figura 1. La selección del método docente.

Fuente: La selección del método docente. (Fernández March , 2005)

Según Fernández March (2005: p.18), no existe “el mejor método de enseñanza”. Pero aquellos métodos de enseñanza mayoritariamente centrados sobre los estudiantes han demostrado generar más aprendizaje que los métodos centrados en el profesor. Esta es la razón por la que se ha optado por una combinación de distintos métodos, aprovechando las funciones del proceso de enseñanza-aprendizaje que proporciona cada método.

Toda esta combinación de métodos tiene como fin, evitar que el profesor tenga una función únicamente de enseñar. Implica una vocación de enseñar a aprender, de ser guía tanto profesional como personal, en definitiva ser un Coach. Se trata, por tanto, de dar un papel más activo al alumno, favoreciendo su iniciativa y responsabilidad de forma similar a como se le va a exigir posteriormente en el mundo laboral.

Para ello se usan los métodos y los sistemas de evaluación que se detallan a continuación.

\subsection{Lecciones magistrales}

Se definen como la estrategia metodológica basada en la exposición estructurada de un tema. Su finalidad es:

a) transferir información actualizada y bien organizada procedente de diversas fuentes

b) facilitar la comprensión y aplicación de los procedimientos específicos

c) elevar la motivación de los estudiantes hacia la asignatura.

En este caso, se prefiere usar el término “Lección Magistral Participativa” empleado 
Como indican Birenbaum et al. (Birenbaum et al, 2006), el sistema de evaluación tradicional se considera un punto final en vez de un punto de inicio del aprendizaje. Es decir, está más centrado en la evaluación del aprendizaje (reproducción del conocimiento) en lugar de la evaluación para el aprendizaje (transformación del conocimiento).

En las nuevas condiciones que se dan con el EEES, los profesores deben adoptar un enfoque más centrado en los estudiantes. Así, a la hora de diseñar un módulo se debe "reflexionar sobre lo que queremos que los estudiantes aprendan más que sobre lo que enseñamos" (Norton, 2010).

\subsection{Resolución de ejercicios en el aula}

Siguiendo las premisas de Norton (2010), se incorporan a las lecciones magistrales ejercicios de aplicación práctica (utilizando el método de aprendizaje basado en problemas) que los alumnos deben realizar en clases. Una vez finalizado, uno de ellos, escogido al azar, debe explicarlos al resto de la clase. De esta forma, se fomenta la participación de los alumnos y el empleo del espíritu crítico, al poder realizar comentarios a la exposición de su compañero. Además, al finalizar la clase, estos pequeños ejercicios son entregados al profesor, incorporándolos al proceso de evaluación continua-retroalimentación.

\subsection{Estudio de casos/ análisis de situaciones}

Su finalidad es la adquisición de aprendizajes mediante el análisis de casos reales o simulados. El proceso consiste en presentar un caso concreto, con un guión de trabajo que orienta el proceso. Los alumnos, agrupados en número impar de miembros para facilitar los acuerdos (3 ó 5), deben resolverlo en el tiempo que dura la sesión de trabajo (tiempo suficiente para la resolución siguiendo el guión propuesto, ya que el profesor lo ha evaluado con anterioridad).

Con esta modalidad se pretende que los alumnos se familiaricen con los conocimientos y técnicas objeto de aprendizaje. Se trata de que planteen alternativas de solución, que aprendan a debatir, argumentar, escuchar y respetar a los demás, además de transferir las técnicas de trabajo en grupo.

A medida que el curso avanza, el número de miembros del grupo pasa a ser par (4 ó 6), de tal forma que se intensifica la necesidad de defender sus argumentos internamente para llegar a una única solución.

La evaluación va a estar en función de la participación v la contribución de los 
Con objeto de facilitar el desarrollo de relaciones interpersonales, se pide también que los miembros de los grupos no sean estables, de tal forma que se ven forzados a trabajar profesionalmente con personas con las que tienen mayor y menor afinidad. La experiencia demuestra que, en las sesiones iniciales, el grupo trabaja más en términos creativos (divergir) encontrando múltiples alternativas de solución. A medida que avanza el curso, se observa que son capaces de acortar la fase de divergencia y profundizar más en la fase de convergencia hacia una solución final más elaborada (justificación), auto-organizándose mejor en el reparto de tareas y funciones.

\subsection{Proyectos}

En los proyectos, los alumnos elaboran un trabajo sobre el desarrollo de una de las funciones de una empresa, aplicando cada uno de los contenidos de la materia. La finalidad es la aplicación de las habilidades, capacidades y conocimientos adquiridos. En este caso, se trabaja especialmente las habilidades transversales (trabajo en grupo, razonamiento crítico, autorresponsabilidad...).

Para la realización de los proyectos los alumnos se agrupan de forma libre, en 4 grupos de número de miembros variable, pero siempre tratando de tener miembros impares.

Para la asignación de proyectos a grupos, el docente presenta un caso único que es secuenciado en funciones dentro de esa empresa. Cada grupo debe trabajar en el desarrollo de esa función. El docente actúa en este caso asumiendo el rol de director general de la empresa, fijando la estrategia general. Cada grupo pasa a asumir una de las siguientes partes: marketing-comercialización; producción y logística de aprovisionamiento; inversión y finanzas; recursos humanos y diseño de parámetros de la estructura organizativa.

Cada grupo hace la planificación de su ámbito de responsabilidad. El plan de trabajo que van a seguir sus miembros y la asignación de tareas a realizar por cada uno, conjuntamente con un cronograma, es entregado al profesor. El proyecto se realiza de forma autónoma, estableciéndose puntos de control en las horas de clase destinadas a prácticas.

El procedimiento de evaluación de los proyectos, va a ser una combinación de diferentes métodos, ya que lo que se va a evaluar son competencias muy distintas. Se evalúa, por un lado las habilidades y destrezas de cada alumno, a través del trabajo en grupo, de la toma de decisiones dentro del grupo. También se evalúa las actitudes y valores, como la responsabilidad personal y grupal. Y por otro, a través del trabajo 

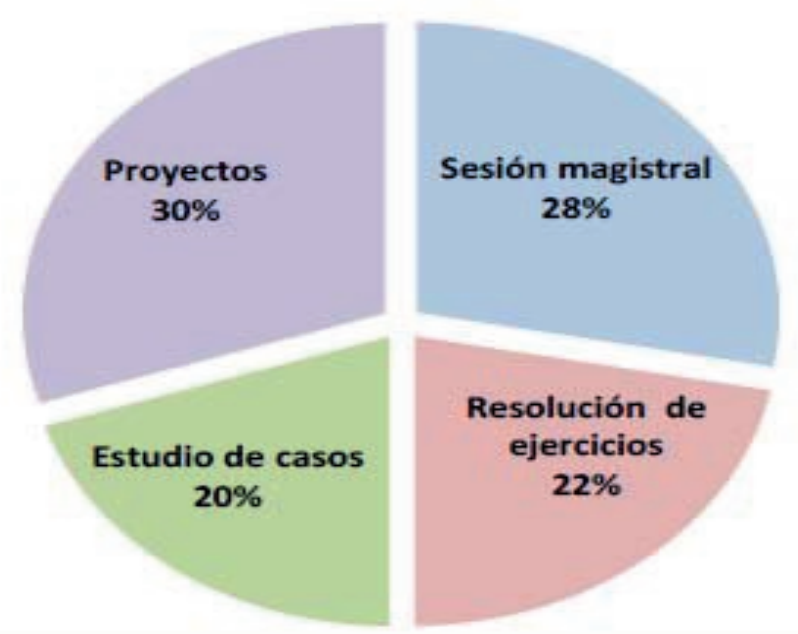

Figura 2. Distribución de la metodología empleada en el módulo.

Fuente: elaboración propia

\subsection{Evaluación del rendimiento: herramientas}

Tal y como se ha descrito, la materia de Economía de la Empresa está repartida de forma que forma que le permita al alumno tener las nociones teóricas necesarias para poder emplearlas en las clases más prácticas, como el estudio de casos y los proyectos. La entrega de ejercicios y del proyecto forma parte de la evaluación continua que se realiza en esta materia, por lo tanto, es valorado en la nota final obtenida.

Desde hace años, se incorpora al comienzo del módulo, una evaluación inicial, para conocer las nociones iniciales sobre la materia y los mitos que tienen sobre ella. De esta forma, se ayuda al alumno a centrar las expectativas que tienen del módulo. Ayuda además a adaptar la estrategia de enseñanza a seguir y los contenidos que deben ser reforzados.

Como complemento a la enseñanza basada en el alumno, se realiza el mismo cuestionario de evaluación al final del módulo. Se trata de ayudar a que el propio alumno se dé cuenta de los conocimientos adquiridos, y sus debilidades para solventarlas antes de la finalización del curso.

Aunque el enfoque seguido es el de potenciar el aprendizaje de forma continua y, por tanto, se debe seguir un método de evaluación continua, es necesario introducir rina nrisoha shiotiva final Fcta nrisha final oc 11 ovamon $\Delta 1$ alımno co lo normito 
a) Conocimientos: no solo conocimientos académicos, a través de las respuestas obtenidas en los ejercicios, sino también en la forma de presentar la solución de forma estructurada. Se valora el razonamiento crítico y a la aplicación de conocimientos adquiridos.

b) Habilidades y destrezas: a través de las estrategias de planificación y gestión del tiempo del examen. Al tener la documentación escrita, la limitación de tiempo para la resolución del examen simula la presión que puedan recibir en situaciones profesionales similares.

c) Actitudes y valores: la prueba final supone una evaluación de sus competencias en términos de madurez profesional. Dado que suelen existir dudas sobre la resolución de los ejercicios, se valora su actitud a la hora de defender su solución con respecto a otras alternativas posibles. Dicho asunto también es tratado en la revisión posterior del examen, donde se les envía un resumen del resultado (aspectos positivos y aspectos mejorables), dando también orientaciones para la mejora.

Los alumnos conocen los criterios de evaluación antes de iniciar el estudio de la asignatura, ya que están descritos en el programa.

Estos criterios son:

a) La nota final será la obtenida en el examen final y, en su caso, podrá ser incrementada hasta en un $20 \%$ en función del trabajo desarrollado, la participación del alumno/a en las clases y, especialmente, las entregas previas a la sesión de las propuestas de resolución de los casos prácticos programados. Será necesario obtener al menos el $45 \%$ de la nota máxima para poder aplicar dicho incremento de hasta un $20 \%$.

b) En el resto de los casos, no se aplicará la regla de la nota incremental.

Como se puede observar, se trata de un sistema de incentivos positivos, pero no negativos.

Se ha dado el caso de personas que, en idéntica situación de nota de examen, unos han alcanzado la valoración positiva (y por lo tanto, aprobando) y otros no. En estos casos, existe una retroalimentación con los alumnos que no han demostrado tener un rendimiento mínimo, para hacerles ver en qué han fallado y corregirlo para la siguiente convocatoria.

\section{CONCLUSIONES}


evaluadas las diferentes competencias de forma continua, adaptando las herramientas y métodos a los objetivos planteados. Estos objetivos son la transferencia no solo de conocimiento, sino también de habilidades, destrezas y actitudes.

Es necesario por tanto una combinación de métodos que permita transferir algo más que meros conocimientos. La combinación descrita y el desarrollo de la función de Coaching del docente, permite que el alumno sea capaz de desarrollarse posteriormente en el mundo profesional, con algo más que conocimientos. Se enfatiza en este caso el "aprender-haciendo" (learning-by-doing) y la retroalimentación continúa al alumno.

Se destaca también el papel relevante que tienen las materias del área de organización de empresas en la transferencia de competencias transversales en los grados de ingeniería. Adicionalmente, se puede apuntar que el uso combinado de plataformas telemáticas de apoyo a la docencia facilita enormemente el seguimiento individualizado del alumno, ya que permite diseñar herramientas de seguimiento para observar en qué medida el alumno ha podido dedicar tiempo suficiente, así como sistemas automatizados de autovaloración del rendimiento.

Como estrategia de futuro, se plantea también el posible uso de foros de debate sobre aspectos de aplicación práctica de contenidos de la materia, ya que el alumno está muy habituado al uso de estas herramientas de redes sociales y puede suponer un elemento motivador y de enganche de alumnos de los primeros cursos universitarios. Existen herramientas basadas en plataformas moodle que facilitan la valoración de sus contribuciones a un foro de debate controlado por el coach, o por ejemplo el uso de foros de sugerencias, o la contribución a la construcción de un glosario. En definitiva, parece que en el futuro, el coach también contará con herramientas telemáticas que facilitarán su labor y asistencia continua, tanto dentro como fuera del aula.

\section{REFRENCIAS}

Birenbaum, M.; Breuer, K.; Cascallar, E., et al. (2006). A Learning Integrated Assessment System. Educational Research Review 1(1): 61-67.

Brown, G. \& Atkins, M. (1988). Effective Teaching in Higher Education. Routledge.

De la Cruz Tomé, Ma A. (2004). Un modelo de lección magistral para un aprendizaje activo y cooperativo. En Cursos y Conferencias de Innovación y Desarrollo Docente. Vigo: Universidad de Vigo. 


\section{Miguel González Loureiro}

Profesor asociado al departamento de Organización de Empresas y Marketing de la Universidad de Vigo. Doctor Europeo en Gestión y Dirección de Empresas. Ha presentado diversos trabajos relacionados congresos científicos internacionales y revistas científicas del área de empresa. Forma parte del equipo de revisores de la Harvard Deusto Business Research y de Intangible Capital. Miembro del equipo de investigación G4plus de la Universidad de Vigo, ha colaborado y participado desde el año 1998 en más de 45 proyectos orientados a la transferencia de resultados para la mejora del sistema empresarial. Pertenece a la: Academia Europea de Dirección y Economía de la Empresa-AEDEM; Asociación Científica de Economía y Dirección de Empresa-ACEDE; Asociación Portuguesa de Empreendedorismo-Empreend. 